This item was submitted to Loughborough's Research Repository by the author.

Items in Figshare are protected by copyright, with all rights reserved, unless otherwise indicated.

\title{
Investigating factors affecting photoconductive microwave switch performance using 3D em simulation
}

PLEASE CITE THE PUBLISHED VERSION

http://dx.doi.org/10.1109/LAPC.2015.7366025

PUBLISHER

(C) IEEE

VERSION

AM (Accepted Manuscript)

\section{PUBLISHER STATEMENT}

(C) IEEE. Personal use of this material is permitted. Permission from IEEE must be obtained for all other uses, in any current or future media, including reprinting/republishing this material for advertising or promotional purposes, creating new collective works, for resale or redistribution to servers or lists, or reuse of any copyrighted component of this work in other works.

\section{LICENCE}

\section{All Rights Reserved}

\section{REPOSITORY RECORD}

Kowalczuk, Emma K., Rob Seager, and Chinthana Panagamuwa. 2019. "Investigating Factors Affecting Photoconductive Microwave Switch Performance Using 3D Em Simulation”. figshare.

https://hdl.handle.net/2134/25910. 


\title{
Investigating Factors Affecting Photoconductive Microwave Switch Performance using 3D EM Simulation
}

\author{
Emma K. Kowalczuk, Rob D. Seager and Chinthana J. Panagamuwa \\ WiCR Group, School of Electronic, Electrical and Systems Engineering, \\ Loughborough University, Leics., U.K.
}

\begin{abstract}
A series of 3D EM simulation models are presented in order to determine the effect that conductivity profile, passivation layer and connection method have on the transmission performance of a photoconductive microwave switch. The use of 3D EM simulation can help quantify the benefit and impact of different approaches before the manufacture stage. The aim is to find methods to reduce insertion loss of the switch to provide maximum efficiency when the device is integrated into reconfigurable applications. Results show improvement to the transmission is possible by altering passivation thickness and designing optical feed to maintain signal planarity.
\end{abstract}

Keywords-Photoconductive microwave switch, EM simulation, passivation, signal planairty, reconfigurable antenna

\section{INTRODUCTION}

With an ever increasing number of antennas and circuits being integrated into RF systems, the design of reconfigurable circuits has become a necessity. Such systems allow multifunctionality, whilst still conforming to the requirement for a small form factor. Realising such reconfigurability requires the selection and integration of a switching mechanism such as a PIN diode, MEMS switch or photoconductive switch. The performance of the system will be dependent on the switch characteristics, such as effect on radiation patterns due to biasing lines and insertion and isolation which ultimately affect system efficiency.

In this study, the photoconductive switch (PCS), consisting of a silicon die mounted across a gap in transmission line, is investigated. The switch is activated by illuminating the silicon with near infra-red light (wavelength $980 \mathrm{~nm}$ ). This switching method has the advantage that control of the state can be via a fibre optic cable having no effect on the radiation pattern of the antennas. In order to maximise the performance of the switch it is vital to understand the potential conductivity, the most accurate way to simulate the switch and the performance in terms of loss, linearity [1] and switching time. The switch must also be packaged appropriately for easy integration with minimum degradation to overall system performance.

In order to investigate packaging techniques and optimise switch performance, this study demonstrates the factors affecting switch performance such as conductivity profile generated in the silicon and presence of a passivation layer and quantifies them in terms of insertion loss. ON state transmission performance is generated from a 3D EM simulation model for the switch and compared to a measured result in order to improve the simulation model accuracy and identify areas for further enhancement. The advantages to using 3D EM simulation is that the effect that individual elements have on the result is easily quantified in a controlled environment.

When designing reconfigurable circuits or antennas, it is fairly common to simulate the switching element as an 'ideal' component [2]. In the $\mathrm{ON}$ state, this means simulating the antenna with a piece of Perfect Electrical Conductor (PEC), which has infinite conductivity across the gap. In the OFF state this means simulating the design with an open gap (with no bridging material). Modelling the switch in such a way in an antenna system reduces simulation time and complexity, whilst still allowing a proof of concept for the antenna.

This method does not represent the losses in the switch and how it may affect the performance of the antenna. Potential methods for simulating the photoconductive switch loss have been suggested such as the use of equivalent circuits by Bhadauria et al. [3]. Rene et al. [4] add further complexity by considering the current density caused by the generation of carriers under illumination. This is realised as 6 layered hemispherical structure of varying conductivities, simulated using 3D EM simulation tools.

The work presented here also investigates the conductivity profile of the silicon with a focus on how it can be exploited to maintain signal planarity and quantify improvement of transmission performance in the ON state.

\section{SiMULATION MODEL CASES}

Both silicon quality and transmission line topology of the switch dictates insertion loss and isolation of the switch. The topology must be designed in relation to the light source and silicon size, and has been optimised and measured in previous work [5]. This topology has been used here to further improve the simulation model for the PCS to better understand how performance can be enhanced.

The topology comprises two segments of transmission line with a $1 \mathrm{~mm} \times 1 \mathrm{~mm} \times 0.3 \mathrm{~mm}$ block of representative silicon 
material (which will be altered) centred over the gap between transmission lines. Silver epoxy is also included in the simulation model and is modelled as having a conductivity of $20000 \mathrm{~S} / \mathrm{m}$. CST Microwave Studio 2013 [6] is used to conduct the analysis, with waveguide port excitations. The substrate comprises of Rogers 3003 with a thickness of 0.5 $\mathrm{mm}, \varepsilon_{\mathrm{r}}=3, \tan \delta=0.0031$. Transmission line width is $1.2 \mathrm{~mm}$, the length of the line is $30 \mathrm{~mm}$ and the gap between transmission lines is $0.3 \mathrm{~mm}$.

Four cases are investigated using this topology via simulation and are compared to the measured performance of the switch:
a) Perfect switch
b) 6 Layer model
c) 6 Layer model including passivation effect
d) 6 Layer model considering connector

In this work, the transmission in the $\mathrm{ON}$ state is the main focus, however reference to OFF state performance is reported by Kowalczuk in other studies [7]

\section{A. Perfect Conductor}

In reality, no switch is perfect or ideal - each will have an associated insertion loss and isolation and these have an effect on the overall antenna or microwave circuit performance. An ideal switch has $50 \Omega$ impedance in the ON state and infinite impedance in the OFF state.

Firstly, an ideal switch, Fig. 1, is simulated and compared to measured data of the silicon switch. The ON state is replicated by defining the 'switch' block to be a PEC.

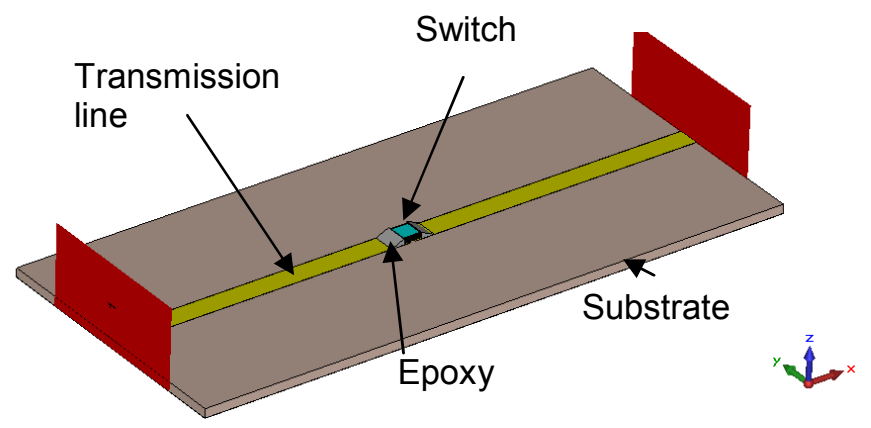

Fig. 1 CAD model of the optically switched transmission line

\section{B. 6 Layer Model}

A more realistic representation of the switch is to replace the PEC block with a set of 6 dielectric materials with varying permittivity and conductivity to more accurately capture the loss. Conductivity within the silicon is increased when the switch is illuminated with near IR light with a wavelength of $980 \mathrm{~nm}$ - the energy of the photons at this wavelength is sufficient to promote the generation of free carriers in the silicon. According to the Beer-Lambert law [8], light intensity decreases exponentially as it penetrates deeper into the silicon, hence giving rise to a changing distribution of free carriers and associated conductivity profile.

The density and distribution of free carriers in a semiconductor is determined by three factors:
- Generation Rate (affected by photon flux and absorption)

- Recombination Rate (affected by silicon quality)

- Diffusion Rate (affected by silicon quality and absorption)

The focus of this paper is to demonstrate how simulation can be used as a tool to quantify how conductivity profile affects insertion loss performance. Hence in the interest of brevity, the detailed mathematical calculations governing these factors are not presented in this paper. Please refer to [7] for full details and the process of factoring in the Auger recombination effect. To summarise, Platte's formula on photoconductivity [9] is used in this study to calculate the number of carriers at increasing depth into the silicon die as presented in Fig. 2. Float zone grown Phosphorous doped silicon is used with a resistivity of $\sim 1.4 \times 10^{6} \Omega \cdot \mathrm{cm}^{-1}$. A passivation layer is grown to improve the carrier lifetime and to act as an anti-reflective coating, reducing reflection of incoming illumination at $980 \mathrm{~nm}$ to $15 \%$ and hence maximising the number of photons absorbed in the silicon.

The carrier lifetime is calculated by taking into account silicon quality and Auger recombination. The silicon quality is poorer at the surface and edges compared with the bulk silicon due to the silicon being diced into samples using a diamond saw. The Auger recombination term can be calculated based on equations from Sinton et al. [10].

As number of carriers increases above $\sim 10^{16} \mathrm{~cm}^{-1}$, the Auger recombination term becomes dominant over other types of recombination. This drastically limits potential conductivity for higher levels of applied photon flux as presented in Fig. 2. One of the advantages to using Platte's equation is that the conductivity profile can be determined for varying wavelengths of light for varying radiant flux values.

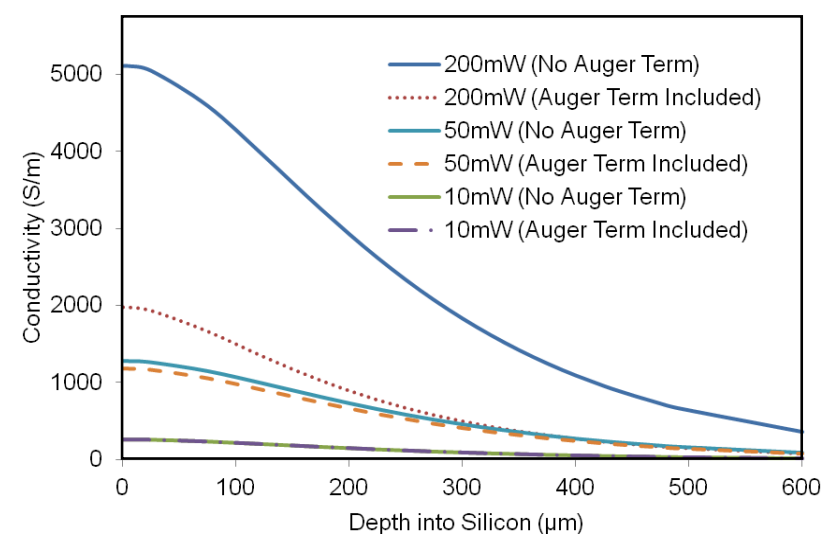

Fig. 2 Conductivity profile for $10 \mathrm{~mW}, 50 \mathrm{~mW}$ and $200 \mathrm{~mW}$ of radiant flux at $980 \mathrm{~nm}$. The graph demonstrates the effect the Auger term has on the conductivity profile.

The number of carriers also affects permittivity of the silicon. Lee et al. [11] present these changes in permittivity as equations which are used in conjunction with Platte's equations to describe six silicon material definitions in the 3D EM simulation tool to represent the changing conductivity and 
permittivity in the die. The theory is described fully in [7], and each layer is $50 \mu \mathrm{m}$ thick, shown in Fig. 3. The corresponding values for conductivity and permittivity at $2 \mathrm{GHz}$ are presented in Table 1 for a radiant flux value of $10 \mathrm{~mW}$.

Since the permittivity is frequency dependent, user defined dispersive materials are defined and the values for permittivity are also calculated and entered as material properties into the EM simulation tool at $1 \mathrm{GHz}, 2 \mathrm{GHz}, 4 \mathrm{GHz}, 8 \mathrm{GHz}$ and 10 $\mathrm{GHz}$. An automatic fitting scheme is applied to determine the values in between.

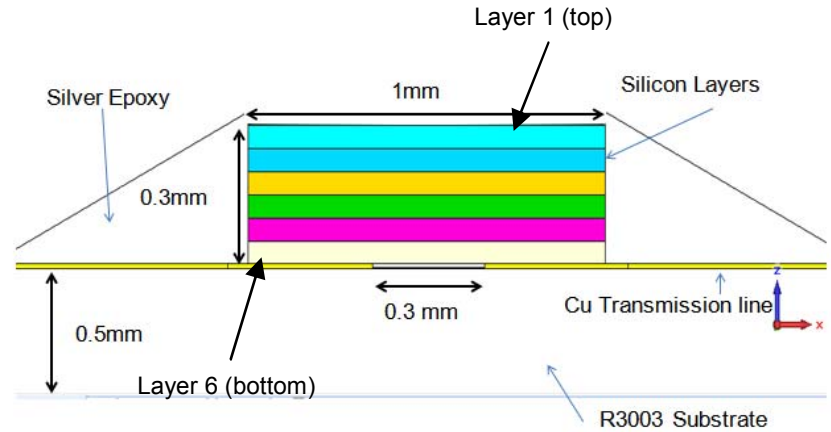

Fig. 36 Layer simulation model of silicon including silver loaded epoxy on a Rogers 3003 substrate with dimensions

TABLE I. MATERIAL DEFINITION AT $2 \mathrm{GHZ}$ FOR RADIANT FLUX OF $10 \mathrm{MW}$

\begin{tabular}{|c|c|c|c|}
\hline Layer no. & $\boldsymbol{\sigma} \mathbf{( S / m )}$ & $\boldsymbol{\varepsilon}_{\mathbf{r}}{ }^{\mathbf{1}}$ & $\boldsymbol{\varepsilon}_{\mathbf{r}}{ }^{\mathbf{I}}$ \\
\hline 1 (top) & 259 & 7.33 & 1749 \\
\hline 2 & 236 & 7.74 & 1593 \\
\hline 3 & 203 & 8.33 & 1368 \\
\hline 4 & 168 & 8.94 & 1132 \\
\hline 5 & 135 & 9.51 & 913 \\
\hline 6 (bottom) & 107 & 10.01 & 723 \\
\hline
\end{tabular}

\section{6 Layer Model-Passivation}

To improve the carrier lifetime of the silicon, a passivation layer is often grown in order to repair dangling bonds on the surface. These dangling bonds are created during the fabrication process, and are sites of high recombination in the silicon, hence limiting the conductivity of the silicon die. The passivation layer is a layer of insulating silicon dioxide, which in photoconductive applications can also serve as an antireflective coating.

For this simulation model case the layered block of silicon is modeled as the 6 layer profile with a $200 \mathrm{~nm}$ thick layer of vacuum around the whole silicon die. The simplification of a vacuum material reduces simulation time, but determines the affect on the S-parameter response

\section{6 Layer Model-Passivation and connector}

Lastly the effect of the SMA connector is modelled by including the SMA geometry, which is generated using Antenna Magus [12] and imported into the CST Microwave
Studio project, Fig. 4. A waveguide port is used to excite the SMA aperture directly, ensuring the correct TEM mode is injected into the structure. This addition of the connector geometry will increase the simulation time due to the added complexity, however it allows the effect of the connectors to be properly quantified.

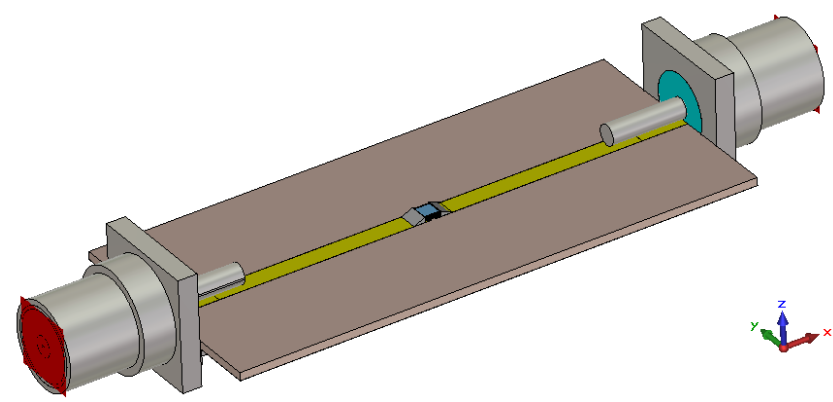

Fig. 4 CAD model of the optically switched transmission line with SMA connectors

\section{SimUlation RESULtS}

Measured results in Fig. 5 demonstrate that depending on radiant flux applied, the transmission through the silicon differs. For the maximum radiant flux used $(200 \mathrm{~mW})$, the ideal switch simulation and measured results differ, demonstrating an extra $8.6 \%$ power loss. The loss is further increased when considering the lower radiant flux value of $10 \mathrm{~mW}$. The difference in $\mathrm{S} 21$ for the measured $10 \mathrm{~mW}$ radiant flux case and simulated PEC switch is $1.75 \mathrm{~dB}$. If using this level of illumination, then it is not accurate to assume an ideal switch using PEC as the PCS material.

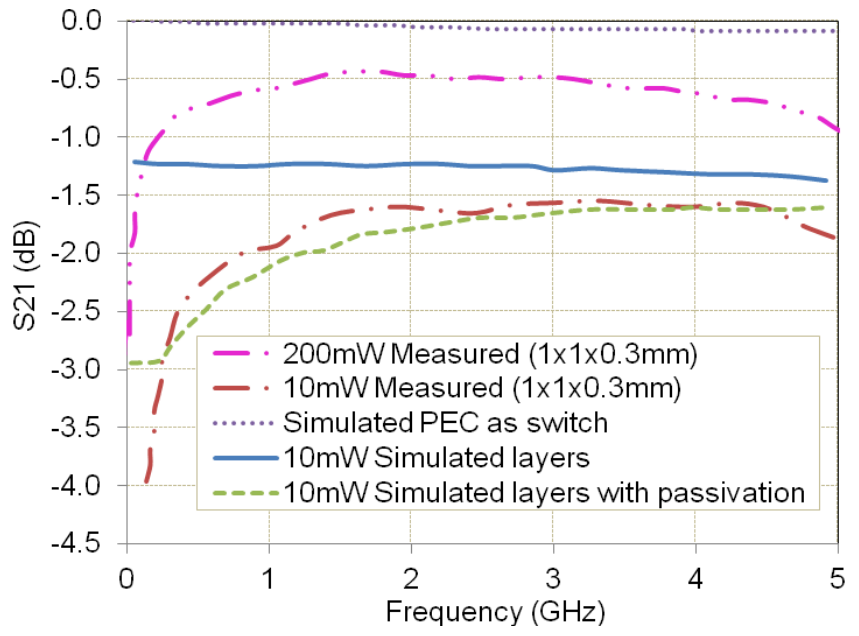

Fig. 5 PCS simulation results in terms of transmission alongside measured $\mathrm{S} 21$ results for various values of radiant flux

The 6-layer representation better captures the loss in the switch and matches the measured result more closely. Different levels of radiant flux can be modeled by adjusting the layer properties- in this study a radiant flux of $10 \mathrm{~mW}$ is assumed. Difference in insertion loss is $0.6 \mathrm{~dB}$ at $2 \mathrm{GHz}$ between measured $10 \mathrm{~mW}$ radiant flux result and 6 layer simulated case. 
However the low frequency performance is not represented accurately due to the lack of passivation layer. When the 200 $\mathrm{nm}$ layer of vacuum is included, the correlation of insertion loss is improved across the band, but is particularly improved for frequencies below $1 \mathrm{GHz}$.

Hence, it can be observed that the decrease in transmission towards DC can be attributed to the presence of oxide. The passivation interferes with the $\mathrm{Si}-\mathrm{Cu}$ contact, leading to a poor connection between the die and transmission line. The passivation layer is not applied to the sawn edge faces of the die, however, native oxide naturally grows on these sawn surfaces. The edges also have a high density of recombination sites due to the poor quality caused by dicing the silicon. Hence very low conductivity is expected at the edge region.

Simulation results show that the insertion loss of the switch can be reduced if the passivation layer is not present or if the layer is reduced in thickness allowing better capacitive coupling of the RF energy to the silicon die from the copper transmission line. Removing the passivation entirely is not feasible as it would result in dangling bonds which reduce the effective carrier lifetime, which in turn limits the number of free carriers.

The layer also acts as an anti-reflective coating in order to maximise light transmission into the silicon and hence ideally is required to be $\sim$ quarter of a wavelength thick with regards to the illumination wavelength. This thickness of passivation is only required on the area that is illuminated by the laser. Elsewhere the passivation layer can potentially be reduced in thickness to allow maximum coupling of RF energy into the die, hence improving lower frequency behaviour of the switch. In addition, the sawn edges of the die greatly reduce the effective carrier lifetime. Selectively doping the two end edges to create ohmic contacts, whilst maintaining passivation on the top and bottom surface of the die, would more easily facilitate signal transmission.

The last case investigates the effect of the connectors - where it is apparent that the connector geometry contributes to the insertion loss parameters as shown in Fig. 6.

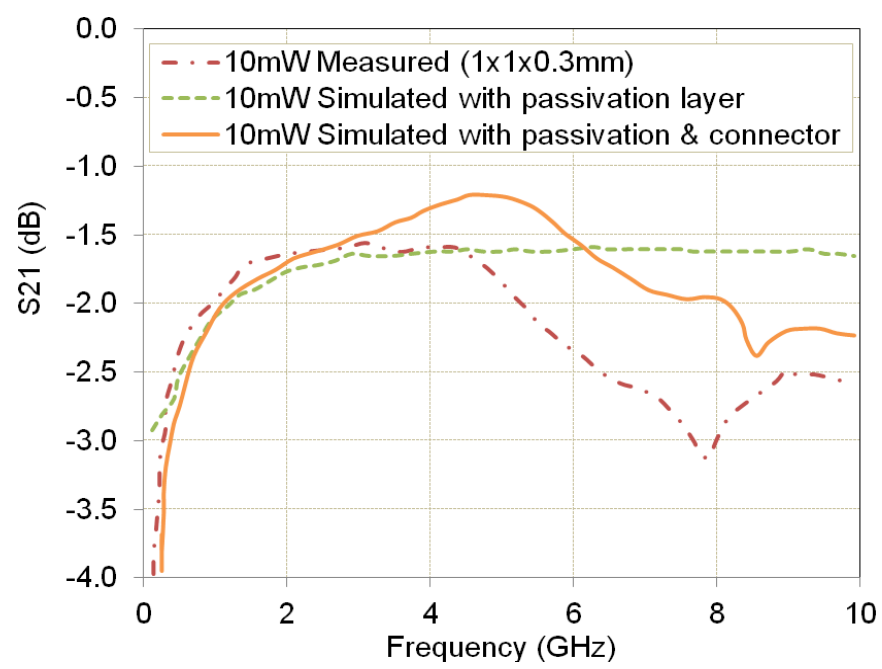

Fig. 6 PCS simulation results including passivation and connector in terms of transmission alongside measured $\mathrm{S} 21$ results for $10 \mathrm{~mW}$ radiant flux
Up to $5 \mathrm{GHz}$, the connectors have a small influence on the insertion loss, a $0.2 \mathrm{~dB}$ decrease when comparing simulation results with and without the connectors at $2 \mathrm{GHz}$. However, if the frequency range is extended, the connectors have a greater affect on the loss. Between $5-8 \mathrm{GHz}$ the connectors add additional loss to the system, $0.65 \mathrm{~dB}$ at $10 \mathrm{GHz}$ which can also be observed in the measured result. There is some discrepancy been the S21 values between simulation and measured data, which may be caused by the unknown dielectric properties and exact geometry of the SMA connector.

Typically connectors are designed to be low loss components, however in reality there will always be some loss of signal and interaction of the connector housing and signal propagating along the cable from the test set. The loss from cables and adaptors can be removed using calibration techniques, however it is more difficult to ascertain the affect of the connector on PCB board. When looking at small differences in insertion loss, the connector may contribute to this value, as is documented in a study performed by CST comparing simulations to measurements [13]. If the bandwidth of interest is below $5 \mathrm{GHz}$ the influence of the connectors could be ignored to speed up the simulation time to make further analysis more productive.

\section{SIGNAL PlanARITY}

So far, illumination has been assumed to be from above the silicon die. However simulation allows a number of scenarios to be investigated with the aim of improving performance. Since it is established that conductivity is highest closest to the illumination source, it can be advantageous to have this surface of the silicon die in contact with the copper transmission line in order to improve signal planarity.

In this preliminary study, the practicalities of illuminating the silicon die from below will be ignored. The motive behind this investigation is to determine the benefit of this approach before moving on to design and fabrication stage. The simulation model used includes the connectors and passivation layer for maximum accuracy with the order of the silicon layers reversed as in Fig. 7.

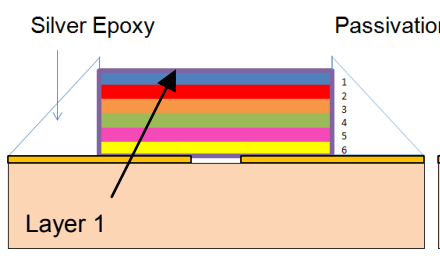

(a)

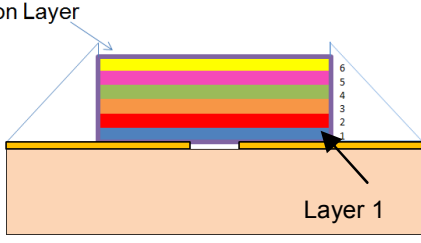

(b)
Fig. 7 Layered silicon model including silver epoxy on a Rogers 3003 substrate with a passivation coating- illumination is from above (a) and below (b) - diagram is not to scale

Fig. 8 compares the silicon illuminated from below and above cases. Of particular interest is the transmission in the ON state. Here, it is clear that the transmission is only marginally improved by choosing to place the highest conductivity layer closest to the transmission line. At $2 \mathrm{GHz}$, the improvement is $0.18 \mathrm{~dB}$. This corresponds to an $\mathrm{S} 21$ of $-1.55 \mathrm{~dB}$ for the silicon 
die illuminated from below and, $-1.73 \mathrm{~dB}$ for the die illuminated from above. At $10 \mathrm{GHz}$ the improvement is 0.25 $\mathrm{dB}$. The corresponding S21 at this frequency for the die illuminated from below is $-1.95 \mathrm{~dB}$.

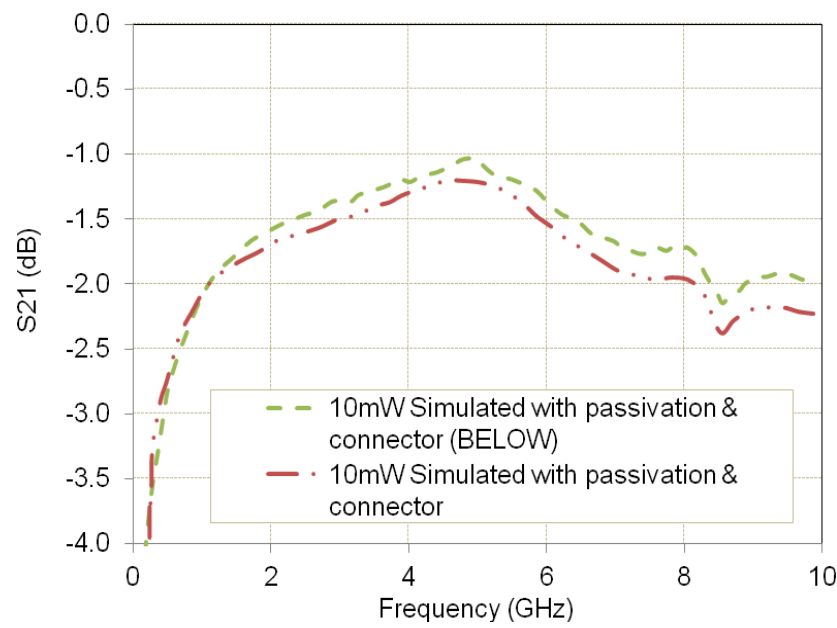

Fig. 8 S21 performance of optically controlled transmission line with silicon illuminated from above and below. Measured result corresponds to illumination from above.

Although the physical optical feed is not considered here, it can be noted that there is some small benefit from having the most conductive area of the silicon in close proximity to the copper transmission line, hence maintaining signal planarity. In order to realise such a scenario, the use of transparent substrates and conductors such as indium tin oxide could also enable feeding from below the substrate a feasible option as reported in [14].

The limited improvement in insertion loss can be attributed to two reasons. Firstly, the difference is conductivity between top and bottom layer is not particularly large for a $10 \mathrm{~mW}$ radiant flux. There is $107 \mathrm{~S} / \mathrm{m}$ conductivity on the layer furthest from the illumination, compared to the top layer of $259 \mathrm{~S} / \mathrm{m}$.

If a shorter wavelength of light is used the penetration depth is reduced. The conductivity profile would have a much larger variation, where the highest conductivity is concentrated at the surface nearest to the illumination. In this case, signal planarity could be more important. This is an area of further work.

The second reason is related to the large skin depth. For the maximum conductivity layer of $259 \mathrm{~S} / \mathrm{m}$ the skin depth is $0.70 \mathrm{~mm}$ at $2 \mathrm{GHz}$ and is $0.31 \mathrm{~mm}$ at $10 \mathrm{GHz}$. The depth of the die is $0.3 \mathrm{~mm}$; hence the skin depth at the highest frequency being investigated and at the highest conductivity being simulated is larger than the thickness of the silicon. This means the signal travels throughout the volume of the silicon, and is not confined to the edges as is the case for high conductivity materials such as Copper. The average conductivity of the illumination from above and below case is the same, and hence the S-parameter performance may be expected to be similar.

\section{CONCLUSION}

A number of different 3D EM simulation models have been investigated in order to ascertain a better correlation between measurements and simulations of a photoconductive microwave switch. The simple PEC representation of the silicon has been replaced with a 6 layered dielectric material approach which is based on silicon properties and is defined to be dispersive in nature. The finite conductivity calculated more accurately defines the loss in the switch. The affect passivation layer and SMA connectors has on insertion loss is also presented individually.

The passivation layer is seen to increase loss particularly at the lower frequencies, hence to improve performance the layer should be reduced in thickness in regions it is not essential. Better signal planarity can be achieved by illuminating the silicon on the surface closest to the copper transmission line, with the result that transmission is improved.

\section{REFERENCES}

[1] E. K. Kowalczuk, C. J. Panagamuwa, R. D. Seager, and J. C. Vardaxoglou, "Characterising the linearity of an optically controlled photoconductive microwave switch," Loughborough Antennas and Propagation Conference, 2010

[2] B-Z. Wang, S. Xiao, and J. Wang. "Reconfigurable patch-antenna design for wideband wireless communication systems." IET Microwaves, Antennas \& Propagation, Vol. 1, no. 2, pp. 414-419, 2007

[3] A. Bhadauria, A. K. Verma, E. K. Sharma, and B. R. Singh. "Optically controlled microstrip load and stub on silicon substrate." Microwave and Optical Technology Letters 39, no. 4, pp. 271-276, 2003.

[4] G. Rene, J. D. Arnould, and A. Vilcot. "Semi-analytical computation and 3D modelling of the microwave photo-induced load in CPW technology." Microwave and optical technology letters 48, no. 9 pp. 1718-1721, 2006.

[5] E. K. Kowalczuk, R. D. Seager, C. J. Panagamuwa, K. Bass and J. C. Vardaxoglou, "Optimising the performance of an optically controlled photoconductive microwave switch," IEEE Loughborough Antennas and Propagation Conference (LAPC), 2012

[6] CST STUDIO SUITE 2013, http://www.cst.com/

[7] E.K. Kowalczuk "Simulating, fabricating and characterising photoconductive microwave switches for RF applications." PhD Thesis, Loughborough University, 2014

[8] R. H. Bube, "Photoelectronic properties of semiconductors" Cambridge University Press, 1992.

[9] W. Platte, and B. Sauerer, "Optically CW-induced losses in semiconductor coplanar waveguides" IEEE Trans. Microwave theory and Techniques, Vol. 37 pp. 139, Jan 1989

[10] R. A. Sinton and R. M. Swanson, "Recombination in highly injected silicon (solar cells)," IEEE Trans. Electron Devices, vol. 34, no. 6, pp. 1380-9, Jun 1987

[11] C. H. Lee, P.S. Mak, and A.P. DeFonzo," Optical control of Millimeterwave propagation in dielectric waveguides," IEEE Journal of quantum electronics, Vol. 16, No. 3, March 1980

[12] Antenna Magus, version 4.3, http://www.antennamagus.com/

[13] M. Rutschlin "Measurement and Simulation in Modern Device Design" Computer Simulation Technology European User Conference, session $5.1 .3,2012$

[14] R. L. Haupt “An Adaptive Rectangular Microstrip Patch Antenna Array Element Using Photonic Controls," IEEE Aerospace Conference, pp. 16, March 2008 